\title{
Stabilization of a laminated beam with interfacial slip by boundary controls
}

\section{Nasser-Eddine Tatar ${ }^{*}$}

\author{
"Correspondence: \\ tatarn@kfupm.edu.sa \\ Department of Mathematics and \\ Statistics, King Fahd University of \\ Petroleum and Minerals, Dhahran, \\ 31261, Saudi Arabia
}

\begin{abstract}
We consider two identical beams on top of each other with an adhesive in between. A considerable natural slip occurs in the structure and will not be ignored as was done in the previous investigations. In this work we take into account this slip and prove that we can stabilize the system in an exponential manner using boundary controls. The model consists of three coupled equations. The first two are related to the well-known Timoshenko system, and the third one describes the dynamic of the slip. Our result improves the few existing similar works in the literature.
\end{abstract}

MSC: 34B05; 34D05; 34H05

Keywords: exponential stabilization; vibration reduction; Timoshenko system; slip dynamic; boundary control; multiplier technique

\section{Introduction}

Of concern is a structure of two identical beams of uniform thickness stuck together by an adhesive. They are placed on top of each other. The structure is subject to a longitudinal displacement in addition to transversal and rotational displacements. These vibrations are undesirable, and it is our goal to stabilize the system in a fast way. These structures have gained much in popularity and are known under the name of 'laminated' beams. They are of considerable importance in engineering. The beams are attached together in such a way that a 'slip' is permitted while they are continuously in contact with each other. In certain situations the slip is purposively allowed with the objective to obtain some damping. This damping should be able to restore the system to its equilibrium state. Fastening very tightly the layers can affect anormally the performance of the structure.

A model based on the Timoshenko theory was derived in [1] (see also [2] for another one), namely

$$
\left\{\begin{array}{l}
\rho w_{t t}+G\left(\psi-w_{x}\right)_{x}=0 \\
I_{\rho}\left(3 s_{t t}-\psi_{t t}\right)-G\left(\psi-w_{x}\right)-D\left(3 s_{x x}-\psi_{x x}\right)=0 \\
3 I_{\rho} s_{t t}+3 G\left(\psi-w_{x}\right)+4 \gamma s+4 \beta s_{t}-3 D s_{x x}=0
\end{array}\right.
$$

where $x \in(0,1)$ and $t>0$, with initial data

$$
(w, \psi, s)(x, 0)=\left(w_{0}, \psi_{0}, s_{0}\right), \quad\left(w_{t}, \psi_{t}, s_{t}\right)(x, 0)=\left(w_{1}, \psi_{1}, s_{1}\right), \quad x \in(0,1)
$$

(c) 2015 Tatar. This article is distributed under the terms of the Creative Commons Attribution 4.0 International License (http://creativecommons.org/licenses/by/4.0/), which permits unrestricted use, distribution, and reproduction in any medium, provided you give appropriate credit to the original author(s) and the source, provide a link to the Creative Commons license, and indicate if changes were made. 
and the cantilever boundary conditions. Here $w, \psi, \rho, G, I_{\rho}, D, \gamma, \beta$ denote the transverse displacement, rotation angle, density, shear stiffness, mass moment of inertia, flexural rigidity, adhesive stiffness and adhesive damping parameter, respectively, and $s$ is proportional to the amount of slip along the interface. It is rather the third equation which is in the spotlight as when $s \equiv 0$ we recover the standard Timoshenko system. As is well known by now, the Timoshenko system has been studied by many authors, and many results may be found in the literature. It has been stabilized by means of different controls such as internal and/or boundary frictional and/or viscoelastic damping, dynamic boundary conditions, pointwise damping, distributed damping, heat damping, etc. The large number of citations cannot fit in this small paper. We refer the reader, however, to [3-12] for similar boundary controls to the ones used here and also for the interesting papers [13-15].

In [16] an exponential decay result was proved for this problem with one end fixed $(w(0, t)=\psi(0, t)=s(0, t)=0, t>0)$ and $\psi(1, t)-w_{x}(1, t)=u_{1}(t), s_{x}(1, t)=0,\left(3 s_{x}-\psi_{x}\right)(1, t)=$ $u_{2}(t), t>0$ at the other end. They adopted the boundary control

$$
u_{1}(t)=k_{1} w_{t}(1, t), \quad u_{2}(t)=-k_{2}\left(3 s_{t}-\psi_{t}\right)(1, t)
$$

and assumed that $r_{1}:=\frac{G}{\rho} \neq \frac{D}{I_{\rho}}=: r_{2}$ and $k_{i} \neq r_{i}, i=1,2$. Moreover, they checked that the damping present in the third equation alone is not able to stabilize the structure in an exponential manner.

Under different boundary controls, namely

$$
\psi(0, t)-w_{x}(0, t)=-k_{1} w_{t}(0, t)-w(0, t), \quad 3 s_{x}(1, t)-\psi_{x}(1, t)=-k_{1} \xi_{t}(1, t)-\xi(1, t),
$$

the authors in [17] proved an exponential stabilization of the system provided that the 'dominant' part of the closed loop system is itself exponentially stable.

Since then, it seems that the subject remained dormant. We would like to bring back to life this matter of treating, in a more adequate fashion, structures for which the amount of slip is considerable.

We discuss here the same model and boundary conditions as in [16]. We prove an exponential stabilization result without assuming the conditions $r_{1}:=\frac{G}{\rho} \neq \frac{D}{I_{\rho}}=: r_{2}$ and $k_{i} \neq r_{i}$, $i=1,2$. These conditions are dropped, and instead we will assume that $\rho G<I_{\rho}$. Our approach is different from the one used in [16]. Namely, we will consider the system

$$
\left\{\begin{array}{l}
\rho w_{t t}+G\left(\psi-w_{x}\right)_{x}=0 \\
I_{\rho}\left(3 s_{t t}-\psi_{t t}\right)-G\left(\psi-w_{x}\right)-(3 s-\psi)_{x x}=0 \\
I_{\rho} s_{t t}+G\left(\psi-w_{x}\right)+\frac{4}{3} \gamma s+\frac{4}{3} \beta s_{t}-s_{x x}=0
\end{array}\right.
$$

for $x \in(0,1), t>0$, with the boundary conditions

$$
\left\{\begin{array}{l}
w(0, t)=\psi(0, t)=s(0, t)=0, \\
\left(\psi-w_{x}\right)(1, t)=k_{1} w_{t}(1, t), \quad s_{x}(1, t)=0, \\
(3 s-\psi)_{x}(1, t)=-k_{2}(3 s-\psi)_{t}(1, t)
\end{array}\right.
$$

for $t \geq 0$. 
The well-posedness follows easily from a slight modification of the arguments in $[16,17]$ (see also references in [1]). We have weak solutions in $\left(V_{*}^{1} \times L^{2}\right)^{3}$ and strong solutions in $\left(V_{*}^{2} \times H^{1}\right)^{3}$, where

$$
V_{*}^{k}=\left\{v: v \in H^{k}(0,1): v(0)=0\right\}, \quad k=1,2
$$

We shall focus here on the asymptotic behavior of solutions and in particular on the exponential stabilization of the system.

In the next section we prove that the energy is decreasing, define the different functionals we will utilize later and prepare some useful lemmas. Section 2 is devoted to the statement and proof of our main result. The last section is a short one containing our conclusion.

\section{Some useful preliminary results}

The energy of system (1)-(2) is given by

$$
\begin{aligned}
E(t)= & \frac{1}{2}\left[3 I_{\rho}\left\|s_{t}\right\|^{2}+4 \gamma\|s\|^{2}+3\left\|s_{x}\right\|^{2}+I_{\rho}\left\|3 s_{t}-\psi_{t}\right\|^{2}+\left\|(3 s-\psi)_{x}\right\|^{2}\right. \\
& \left.+\rho\left\|w_{t}\right\|^{2}+G\left\|\psi-w_{x}\right\|^{2}\right], \quad t \geq 0,
\end{aligned}
$$

where $\|\cdot\|$ denotes the norm in $L^{2}(0,1)$.

Proposition 1 The energy $E(t)$ is decreasing and in fact we have

$$
E^{\prime}(t)=-G k_{1} w_{t}^{2}(1)-4 \beta\left\|s_{t}\right\|^{2}-k_{2}(3 s-\psi)_{t}^{2}(1), \quad t \geq 0
$$

Proof This follows directly by multiplying the first equation in (1) by $w_{t}$ (the second by $(3 s-\psi)_{t}$ and the third by $\left.s_{t}\right)$ and integrating over $(0,1)$. Integration by parts and the boundary conditions will also be used. Indeed, we obtain

$$
\frac{\rho}{2} \frac{d}{d t}\left\|w_{t}\right\|^{2}+G\left(\left(\psi-w_{x}\right)_{x}, w_{t}\right)=0, \quad t \geq 0
$$

or

$$
\frac{\rho}{2} \frac{d}{d t}\left\|w_{t}\right\|^{2}-G\left(\psi-w_{x}, w_{x t}\right)+\left[G\left(\psi-w_{x}\right) w_{t}\right]_{0}^{1}=0, \quad t \geq 0
$$

and by our boundary conditions

$$
\frac{\rho}{2} \frac{d}{d t}\left\|w_{t}\right\|^{2}-G\left(\psi-w_{x}, w_{x t}\right)+G k_{1} w_{t}^{2}(1)=0, \quad t \geq 0 .
$$

The observation

$$
G\left(\psi-w_{x}, w_{x t}\right)=-G\left(\psi-w_{x},\left(\psi-w_{x}-\psi\right)_{t}\right)=-\frac{G}{2} \frac{d}{d t}\left\|\psi-w_{x}\right\|^{2}+G\left(\psi-w_{x}, \psi_{t}\right)
$$

leads to

$$
\frac{1}{2} \frac{d}{d t}\left[\rho\left\|w_{t}\right\|^{2}+G\left\|\psi-w_{x}\right\|^{2}\right]-G\left(\psi-w_{x}, \psi_{t}\right)+G k_{1} w_{t}^{2}(1)=0, \quad t \geq 0 .
$$


Working with the third equation of (1) we arrive at

$$
I_{\rho}\left(s_{t t}, s_{t}\right)+G\left(\psi-w_{x}, s_{t}\right)+\frac{4 \gamma}{3}\left(s, s_{t}\right)+\frac{4 \beta}{3}\left\|s_{t}\right\|^{2}-\left(s_{x x}, s_{t}\right)=0
$$

or

$$
\frac{1}{2} \frac{d}{d t}\left[I_{\rho}\left\|s_{t}\right\|^{2}+\frac{4 \gamma}{3}\|s\|^{2}+\left\|s_{x}\right\|^{2}\right]+G\left(\psi-w_{x}, s_{t}\right)+\frac{4 \beta}{3}\left\|s_{t}\right\|^{2}=0, \quad t \geq 0 .
$$

Summing up (4) and (5) gives

$$
\begin{aligned}
& \frac{1}{2} \frac{d}{d t}\left[3 I_{\rho}\left\|s_{t}\right\|^{2}+4 \gamma\|s\|^{2}+3\left\|s_{x}\right\|^{2}+\rho\left\|w_{t}\right\|^{2}+G\left\|\psi-w_{x}\right\|^{2}\right] \\
& \quad+G\left(\psi-w_{x}, 3 s_{t}-\psi_{t}\right)+4 \beta\left\|s_{t}\right\|^{2}+G k_{1} w_{t}^{2}(1)=0, \quad t \geq 0 .
\end{aligned}
$$

The second equation in (1) allows us to write, for $t \geq 0$,

$$
\begin{aligned}
\frac{I_{\rho}}{2} \frac{d}{d t}\left\|3 s_{t}-\psi_{t}\right\|^{2} \\
\quad=\left(G\left(\psi-w_{x}\right)+(3 s-\psi)_{x x},(3 s-\psi)_{t}\right) \\
\quad=G\left(\psi-w_{x},(3 s-\psi)_{t}\right)-\left((3 s-\psi)_{x},(3 s-\psi)_{x t}\right)+\left[(3 s-\psi)_{x}(3 s-\psi)_{t}\right]_{0}^{1} \\
\quad=G\left(\psi-w_{x},(3 s-\psi)_{t}\right)-\left((3 s-\psi)_{x},(3 s-\psi)_{x t}\right)-k_{2}(3 s-\psi)_{t}^{2}(1)
\end{aligned}
$$

or

$$
\frac{1}{2} \frac{d}{d t}\left[I_{\rho}\left\|3 s_{t}-\psi_{t}\right\|^{2}+\left\|(3 s-\psi)_{x}\right\|^{2}\right]=G\left(\psi-w_{x},(3 s-\psi)_{t}\right)-k_{2}(3 s-\psi)_{t}^{2}(1) .
$$

Our assertion follows at once from (6) and (7).

Although from this proposition we see that the energy is uniformly bounded and decreasing, it is not clear how to prove exponential decay from this functional at this stage. We need to establish a new functional $F(t)$ which is suitable enough to derive an exponential decay. The strategy (which is by now standard) consists in starting with the energy $E(t)$ and modifying it by adding new adequate terms (functionals) which may be estimated below and above by similar terms already existing in the expression of the energy (leading to the equivalence of both functionals) and whose derivatives provide us with the missing terms in the energy after differentiation. That is, the goal is to obtain an inequality of the form $F^{\prime}(t) \leq-\kappa F(t)$ for some positive constant $\kappa$.

We claim that the functional

$$
F(t):=E(t)+\sum_{i=1}^{5} \delta_{i} L_{i}(t), \quad t \geq 0
$$

with

$$
L_{1}(t)=I_{\rho}\left(s_{t}, s\right)-\rho\left(w_{t}, S\right), \quad L_{2}(t)=I_{\rho}\left(3 s_{t}-\psi_{t}, 3 s-\psi\right),
$$




$$
\begin{aligned}
& L_{3}(t)=-\rho\left(x w_{t}, \psi-w_{x}\right), \quad L_{4}(t)=I_{\rho}\left(x\left(3 s_{x}-\psi_{x}\right), 3 s_{t}-\psi_{t}\right), \\
& L_{5}(t)=-\rho\left(w_{t}, w\right),
\end{aligned}
$$

where

$$
S(x, t)=-\int_{0}^{x} s(r, t) d r
$$

for $\delta_{i}>0, i=1, \ldots, 5$, to be determined, is an appropriate one.

It is easy to see that $F(t)$ and $E(t)$ are equivalent. Simple use of the Cauchy-Schwarz inequality and the Poincaré inequality will do.

Lemma 1 The derivative of $L_{1}(t)$ along solutions of (1)-(2) is estimated by

$$
\begin{aligned}
L_{1}^{\prime}(t) \leq & \left(I_{\rho}+\frac{\rho^{2}}{4 \varepsilon_{0}}+\frac{4 \beta^{2}}{9 \varepsilon_{0}}\right)\left\|s_{t}\right\|^{2}+\left(\varepsilon_{0}-1\right)\left\|s_{x}\right\|^{2}+\left(\varepsilon_{0}-\frac{4 \gamma}{3}\right)\|s\|^{2} \\
& +\varepsilon_{0}\left\|w_{t}\right\|^{2}+\frac{k_{1}^{2} G^{2}}{4 \varepsilon_{0}} w_{t}^{2}(1), \quad t \geq 0, \varepsilon_{0}>0 .
\end{aligned}
$$

Proof As simple differentiation of $L_{1}(t)$ and use of the first and third equations in system (1) yield

$$
\begin{aligned}
L_{1}^{\prime}(t)= & I_{\rho}\left(s_{t}, s_{t}\right)+I_{\rho}\left(s_{t t}, s\right)-\rho\left(w_{t t}, S\right)-\rho\left(w_{t}, S_{t}\right) \\
= & I_{\rho}\left\|s_{t}\right\|^{2}-G\left(\psi-w_{x}, s\right)-\frac{4 \gamma}{3}\|s\|^{2}-\frac{4 \beta}{3}\left(s_{t}, s\right)-\left\|s_{x}\right\|^{2} \\
& +G\left(\left(\psi-w_{x}\right)_{x}, S\right)-\rho\left(w_{t}, S_{t}\right), \quad t \geq 0 .
\end{aligned}
$$

Observe that

$$
\begin{aligned}
G\left(\left(\psi-w_{x}\right)_{x}, S\right) & =G\left[\left(\psi-w_{x}\right) S\right]_{0}^{1}-G\left(\psi-w_{x}, S_{x}\right) \\
& =G\left(\psi-w_{x}\right)(1) S(1)+G\left(\psi-w_{x}, s\right) \\
& \leq \varepsilon_{0}\left\|s_{x}\right\|^{2}+\frac{k_{1}^{2} G^{2}}{4 \varepsilon_{0}} w_{t}^{2}(1)+G\left(\psi-w_{x}, s\right), \quad t \geq 0 .
\end{aligned}
$$

Therefore

$$
\begin{aligned}
L_{1}^{\prime}(t) \leq & \left(I_{\rho}+\frac{\rho^{2}}{4 \varepsilon_{0}}+\frac{4 \beta^{2}}{9 \varepsilon_{0}}\right)\left\|s_{t}\right\|^{2}+\left(\varepsilon_{0}-1\right)\left\|s_{x}\right\|^{2}+\left(\varepsilon_{0}-\frac{4 \gamma}{3}\right)\|s\|^{2} \\
& +\varepsilon_{0}\left\|w_{t}\right\|^{2}+\frac{k_{1}^{2} G^{2}}{4 \varepsilon_{0}} w_{t}^{2}(1), \quad t \geq 0 .
\end{aligned}
$$

Lemma 2 The derivative of $L_{2}(t)$ along solutions of (1)-(2) satisfies

$$
\begin{aligned}
L_{2}^{\prime}(t) \leq & I_{\rho}\left\|3 s_{t}-\psi_{t}\right\|^{2}+\left(\varepsilon_{0}-\frac{1}{2}\right)\left\|(3 s-\psi)_{x}\right\|^{2}+\frac{G^{2}}{2}\left\|\psi-w_{x}\right\|^{2} \\
& +\frac{k_{2}^{2}}{4 \varepsilon_{0}}(3 s-\psi)_{t}^{2}(1), \quad t \geq 0, \varepsilon_{0}>0 .
\end{aligned}
$$


Proof Using the second equation of (1), we find

$$
\begin{aligned}
I_{\rho} \frac{d}{d t}\left(3 s_{t}-\psi_{t}, 3 s-\psi\right) \\
\quad=I_{\rho}\left\|3 s_{t}-\psi_{t}\right\|^{2}+G\left(\psi-w_{x}, 3 s-\psi\right)+\left[\left(3 s_{x}-\psi_{x}\right)(3 s-\psi)\right]_{0}^{1}-\left\|3 s_{x}-\psi_{x}\right\|^{2} \\
\quad \leq I_{\rho}\left\|3 s_{t}-\psi_{t}\right\|^{2}+G\left(\psi-w_{x}, 3 s-\psi\right)+\left(\varepsilon_{0}-1\right)\left\|(3 s-\psi)_{x}\right\|^{2} \\
\quad+\frac{k_{2}^{2}}{4 \varepsilon_{0}}(3 s-\psi)_{t}^{2}(1), \quad t \geq 0 .
\end{aligned}
$$

The estimate

$$
G\left(\psi-w_{x}, 3 s-\psi\right) \leq \frac{1}{2}\left\|(3 s-\psi)_{x}\right\|^{2}+\frac{G^{2}}{2}\left\|\psi-w_{x}\right\|^{2}
$$

implies that

$$
\begin{aligned}
L_{2}^{\prime}(t) \leq & I_{\rho}\left\|3 s_{t}-\psi_{t}\right\|^{2}+\left(\varepsilon_{0}-\frac{1}{2}\right)\left\|(3 s-\psi)_{x}\right\|^{2}+\frac{G^{2}}{2}\left\|\psi-w_{x}\right\|^{2} \\
& +\frac{k_{2}^{2}}{4 \varepsilon_{0}}(3 s-\psi)_{t}^{2}(1), \quad t \geq 0 .
\end{aligned}
$$

Lemma 3 The derivative of the functional $L_{3}(t)$ along solutions of (1)-(2) may be estimated as follows:

$$
\begin{aligned}
L_{3}^{\prime}(t) \leq & \frac{\rho}{2}\left\|\psi_{t}-3 s_{t}\right\|^{2}+\varepsilon_{0}\left\|w_{t}\right\|^{2}+\frac{9 \rho^{2}}{4 \varepsilon_{0}}\left\|s_{t}\right\|^{2}+\frac{\rho+G k_{1}^{2}}{2} w_{t}^{2}(1) \\
& -\frac{G}{2}\left\|\psi-w_{x}\right\|^{2}, \quad t \geq 0, \varepsilon_{0}>0 .
\end{aligned}
$$

Proof In view of the first and third equations in (1) and the definition of $L_{3}(t)$, we see that

$$
\begin{aligned}
L_{3}^{\prime}(t)= & -\rho\left(x w_{t},\left(\psi-w_{x}\right)_{t}\right)-\rho\left(x w_{t t}, \psi-w_{x}\right) \\
= & -\rho\left(x w_{t}, \psi_{t}\right)+\rho\left(x w_{t}, w_{x t}\right)+G\left(x\left(\psi-w_{x}\right)_{x}, \psi-w_{x}\right) \\
= & -\rho\left(x w_{t}, \psi_{t}-3 s_{t}\right)-3 \rho\left(x w_{t}, s_{t}\right)+\frac{\rho}{2}\left(x, \frac{d w_{t}^{2}}{d x}\right)+\frac{G}{2}\left(x, \frac{d\left(\psi-w_{x}\right)^{2}}{d x}\right) \\
= & -\rho\left(x w_{t}, \psi_{t}-3 s_{t}\right)-3 \rho\left(x w_{t}, s_{t}\right)+\frac{\rho}{2}\left[x w_{t}^{2}\right]_{0}^{1}+\frac{G}{2}\left[x\left(\psi-w_{x}\right)^{2}\right]_{0}^{1} \\
& -\frac{\rho}{2}\left\|w_{t}\right\|^{2}-\frac{G}{2}\left\|\psi-w_{x}\right\|^{2}, \quad t \geq 0 .
\end{aligned}
$$

Therefore, from our boundary conditions

$$
\begin{aligned}
L_{3}^{\prime}(t)= & -\rho\left(x w_{t}, \psi_{t}-3 s_{t}\right)-3 \rho\left(x w_{t}, s_{t}\right)+\frac{\rho}{2} w_{t}^{2}(1) \\
& +\frac{G}{2}\left(\psi-w_{x}\right)^{2}(1)-\frac{\rho}{2}\left\|w_{t}\right\|^{2}-\frac{G}{2}\left\|\psi-w_{x}\right\|^{2}, \quad t \geq 0 .
\end{aligned}
$$

Next, the estimations

$$
-\rho\left(x w_{t}, \psi_{t}-3 s_{t}\right) \leq \frac{\rho}{2}\left\|w_{t}\right\|^{2}+\frac{\rho}{2}\left\|\psi_{t}-3 s_{t}\right\|^{2}
$$


and

$$
-3 \rho\left(x w_{t}, s_{t}\right) \leq \varepsilon_{0}\left\|w_{t}\right\|^{2}+\frac{9 \rho^{2}}{4 \varepsilon_{0}}\left\|s_{t}\right\|^{2}
$$

imply that

$$
\begin{aligned}
L_{3}^{\prime}(t) \leq & \frac{\rho}{2}\left\|w_{t}\right\|^{2}+\frac{\rho}{2}\left\|\psi_{t}-3 s_{t}\right\|^{2}+\varepsilon_{0}\left\|w_{t}\right\|^{2}+\frac{9 \rho^{2}}{4 \varepsilon_{0}}\left\|s_{t}\right\|^{2} \\
& +\frac{\rho}{2} w_{t}^{2}(1)+\frac{G}{2}\left(\psi-w_{x}\right)^{2}(1)-\frac{\rho}{2}\left\|w_{t}\right\|^{2}-\frac{G}{2}\left\|\psi-w_{x}\right\|^{2} \\
\leq & \frac{\rho}{2}\left\|\psi_{t}-3 s_{t}\right\|^{2}+\varepsilon_{0}\left\|w_{t}\right\|^{2}+\frac{9 \rho^{2}}{4 \varepsilon_{0}}\left\|s_{t}\right\|^{2} \\
& +\frac{\rho+G k_{1}^{2}}{2} w_{t}^{2}(1)-\frac{G}{2}\left\|\psi-w_{x}\right\|^{2}, \quad t \geq 0 .
\end{aligned}
$$

Lemma 4 Along solutions of (1)-(2), it holds that

$$
L_{4}^{\prime}(t) \leq \frac{I_{\rho}+k_{2}^{2}}{2}\left(3 s_{t}-\psi_{t}\right)^{2}(1)-\frac{I_{\rho}}{2}\left\|3 s_{t}-\psi_{t}\right\|^{2}+\frac{G^{2}}{2}\left\|\psi-w_{x}\right\|^{2}, \quad t \geq 0 .
$$

Proof Clearly

$$
\begin{aligned}
L_{4}^{\prime}(t)= & I_{\rho}\left(x\left(3 s_{x}-\psi_{x}\right)_{t}, 3 s_{t}-\psi_{t}\right)+I_{\rho}\left(x\left(3 s_{x}-\psi_{x}\right), 3 s_{t t}-\psi_{t t}\right) \\
= & \frac{I_{\rho}}{2}\left(x, \frac{d\left(3 s_{t}-\psi_{t}\right)^{2}}{d x}\right)+\left(x\left(3 s_{x}-\psi_{x}\right), G\left(\psi-w_{x}\right)+(3 s-\psi)_{x x}\right) \\
= & \frac{I_{\rho}}{2}\left(3 s_{t}-\psi_{t}\right)^{2}(1)-\frac{I_{\rho}}{2}\left\|3 s_{t}-\psi_{t}\right\|^{2}+G\left(x\left(3 s_{x}-\psi_{x}\right), \psi-w_{x}\right) \\
& +\frac{1}{2}\left(3 s_{x}-\psi_{x}\right)^{2}(1)-\frac{1}{2}\left\|3 s_{x}-\psi_{x}\right\|^{2}, \quad t \geq 0,
\end{aligned}
$$

and therefore

$$
\begin{aligned}
L_{4}^{\prime}(t) \leq & \frac{I_{\rho}}{2}\left(3 s_{t}-\psi_{t}\right)^{2}(1)-\frac{I_{\rho}}{2}\left\|3 s_{t}-\psi_{t}\right\|^{2}+\frac{1}{2}\left\|3 s_{x}-\psi_{x}\right\|^{2} \\
& +\frac{G^{2}}{2}\left\|\psi-w_{x}\right\|^{2}+\frac{1}{2}\left(3 s_{x}-\psi_{x}\right)^{2}(1)-\frac{1}{2}\left\|3 s_{x}-\psi_{x}\right\|^{2}, \quad t \geq 0,
\end{aligned}
$$

or simply

$$
L_{4}^{\prime}(t) \leq \frac{I_{\rho}+k_{2}^{2}}{2}\left(3 s_{t}-\psi_{t}\right)^{2}(1)-\frac{I_{\rho}}{2}\left\|3 s_{t}-\psi_{t}\right\|^{2}+\frac{G^{2}}{2}\left\|\psi-w_{x}\right\|^{2}, \quad t \geq 0 .
$$

Lemma 5 An estimation of the derivative of $L_{5}(t)$ along solutions of (1)-(2) is given by

$$
\begin{aligned}
L_{5}^{\prime}(t) \leq & -\rho\left\|w_{t}\right\|^{2}+\left(2 \varepsilon_{0}+\frac{3 G}{2}\right)\left\|\psi-w_{x}\right\|^{2}+4 \varepsilon_{0}\left[\left\|(\psi-3 s)_{x}\right\|^{2}+9\|s\|^{2}\right] \\
& +G\left\|\psi_{x}\right\|^{2}+\frac{G^{2} k_{1}^{2}}{4 \varepsilon_{0}} w_{t}^{2}(1), \quad t \geq 0, \varepsilon_{0}>0 .
\end{aligned}
$$


Proof It is easy to see, from the first equation in (1) and the boundary conditions, that

$$
\begin{aligned}
L_{5}^{\prime}(t) & =-\rho\left\|w_{t}\right\|^{2}-\rho\left(w_{t t}, w\right)=-\rho\left\|w_{t}\right\|^{2}+G\left(\left(\psi-w_{x}\right)_{x}, w\right) \\
& =-\rho\left\|w_{t}\right\|^{2}+G\left[\left(\psi-w_{x}\right) w\right]_{0}^{1}-G\left(\psi-w_{x}, w_{x}\right) \\
& =-\rho\left\|w_{t}\right\|^{2}+G\left(\psi-w_{x}\right)(1) w(1)-G\left(\psi-w_{x}, w_{x}\right), \quad t \geq 0 .
\end{aligned}
$$

The last two terms in (9) may be estimated as follows:

$$
G\left(\psi-w_{x}\right)(1) w(1) \leq \varepsilon_{0}\left\|w_{x}\right\|^{2}+\frac{G^{2} k_{1}^{2}}{4 \varepsilon_{0}} w_{t}^{2}(1) \leq 2 \varepsilon_{0}\left\|\psi-w_{x}\right\|^{2}+2 \varepsilon_{0}\|\psi\|^{2}+\frac{G^{2} k_{1}^{2}}{4 \varepsilon_{0}} w_{t}^{2}(1)
$$

so

$$
G\left(\psi-w_{x}\right)(1) w(1) \leq 2 \varepsilon_{0}\left\|\psi-w_{x}\right\|^{2}+4 \varepsilon_{0}\left[\left\|(\psi-3 s)_{x}\right\|^{2}+9\|s\|^{2}\right]+\frac{G^{2} k_{1}^{2}}{4 \varepsilon_{0}} w_{t}^{2}(1),
$$

and

$$
\begin{aligned}
G\left(\psi-w_{x}, w_{x}\right) & \leq \frac{1}{2} G\left\|\psi-w_{x}\right\|^{2}+\frac{G}{2}\left\|w_{x}\right\|^{2} \leq \frac{G}{2}\left\|\psi-w_{x}\right\|^{2}+\frac{G}{2}\left[2\left\|\psi-w_{x}\right\|^{2}+2\left\|\psi_{x}\right\|^{2}\right] \\
& \leq \frac{3 G}{2}\left\|\psi-w_{x}\right\|^{2}+G\left\|\psi_{x}\right\|^{2}, \quad t \geq 0 .
\end{aligned}
$$

Taking into account the previous relations, we find

$$
\begin{aligned}
L_{5}^{\prime}(t) \leq & -\rho\left\|w_{t}\right\|^{2}+2 \varepsilon_{0}\left\|\psi-w_{x}\right\|^{2}+4 \varepsilon_{0}\left[\left\|(\psi-3 s)_{x}\right\|^{2}+9\|s\|^{2}\right]+\frac{G^{2} k_{1}^{2}}{4 \varepsilon_{0}} w_{t}^{2}(1) \\
& +\frac{3 G}{2}\left\|\psi-w_{x}\right\|^{2}+G\left\|\psi_{x}\right\|^{2} \\
\leq & -\rho\left\|w_{t}\right\|^{2}+\left(2 \varepsilon_{0}+\frac{3 G}{2}\right)\left\|\psi-w_{x}\right\|^{2}+4 \varepsilon_{0}\left[\left\|(\psi-3 s)_{x}\right\|^{2}+9\|s\|^{2}\right] \\
& +G\left\|\psi_{x}\right\|^{2}+\frac{G^{2} k_{1}^{2}}{4 \varepsilon_{0}} w_{t}^{2}(1), \quad t \geq 0 .
\end{aligned}
$$

\section{Main result}

Using the previous lemmas we obtain the following result.

Theorem 1 For the energy $E$ defined above, there exist two positive constants $K$ and $\kappa_{0}$ such that

$$
E(t) \leq K e^{-\kappa_{0} t}, \quad t \geq 0
$$

provided that $\rho<I_{\rho} / G$.

Proof In view of Lemmas 1-5, we see that

$$
\begin{aligned}
F^{\prime}(t) \leq & -G k_{1} w_{t}^{2}(1)-4 \beta\left\|s_{t}\right\|^{2}-k_{2}(3 s-\psi)_{t}^{2}(1)+\delta_{1}\left(I_{\rho}+\frac{\rho^{2}}{4 \varepsilon_{0}}+\frac{4 \beta^{2}}{9 \varepsilon_{0}}\right)\left\|s_{t}\right\|^{2} \\
& +\delta_{1}\left(\varepsilon_{0}-1\right)\left\|s_{x}\right\|^{2}+\delta_{1}\left(\varepsilon_{0}-\frac{4 \gamma}{3}\right)\|s\|^{2}+\varepsilon_{0} \delta_{1}\left\|w_{t}\right\|^{2}+\frac{\delta_{1} k_{1}^{2} G^{2}}{4 \varepsilon_{0}} w_{t}^{2}(1)
\end{aligned}
$$




$$
\begin{aligned}
& +\delta_{2} I_{\rho}\left\|3 s_{t}-\psi_{t}\right\|^{2}+\delta_{2}\left(\varepsilon_{0}-\frac{1}{2}\right)\left\|(3 s-\psi)_{x}\right\|^{2}+\frac{\delta_{2} G^{2}}{2}\left\|\psi-w_{x}\right\|^{2} \\
& +\frac{k_{2}^{2} \delta_{2}}{4 \varepsilon_{0}}(3 s-\psi)_{t}^{2}(1)+\frac{\delta_{3} \rho}{2}\left\|\psi_{t}-3 s_{t}\right\|^{2}+\varepsilon_{0} \delta_{3}\left\|w_{t}\right\|^{2}+\frac{9 \rho^{2} \delta_{3}}{4 \varepsilon_{0}}\left\|s_{t}\right\|^{2} \\
& +\frac{\left(\rho+G k_{1}^{2}\right) \delta_{3}}{2} w_{t}^{2}(1)-\frac{G \delta_{3}}{2}\left\|\psi-w_{x}\right\|^{2}+\frac{\left(I_{\rho}+k_{2}^{2}\right) \delta_{4}}{2}\left(3 s_{t}-\psi_{t}\right)^{2}(1) \\
& -\frac{I_{\rho} \delta_{4}}{2}\left\|3 s_{t}-\psi_{t}\right\|^{2}+\frac{G^{2} \delta_{4}}{2}\left\|\psi-w_{x}\right\|^{2}-\delta_{5} \rho\left\|w_{t}\right\|^{2}+G \delta_{5}\left\|\psi_{x}\right\|^{2} \\
& +\frac{G^{2} k_{1}^{2}}{4 \varepsilon_{0}} \delta_{5} w_{t}^{2}(1)+\delta_{5}\left(2 \varepsilon_{0}+\frac{3 G}{2}\right)\left\|\psi-w_{x}\right\|^{2}+4 \varepsilon_{0} \delta_{5}\left[\left\|(\psi-3 s)_{x}\right\|^{2}+9\|s\|^{2}\right]
\end{aligned}
$$

or, using

$$
\left\|\psi_{x}\right\|^{2} \leq 2\left\|(\psi-3 s)_{x}\right\|^{2}+18\left\|s_{x}\right\|^{2}
$$

we find

$$
\begin{aligned}
F^{\prime}(t) \leq & {\left[-G k_{1}+\frac{\delta_{1} k_{1}^{2} G^{2}}{4 \varepsilon_{0}}+\frac{\rho+G k_{1}^{2}}{2} \delta_{3}+\frac{G^{2} k_{1}^{2}}{4 \varepsilon_{0}} \delta_{5}\right] w_{t}^{2}(1) } \\
& +\left[-k_{2}+\frac{k_{2}^{2} \delta_{2}}{4 \varepsilon_{0}}+\frac{I_{\rho}+k_{2}^{2}}{2} \delta_{4}\right](3 s-\psi)_{t}^{2}(1) \\
& +\left[-4 \beta+\delta_{1}\left(I_{\rho}+\frac{\rho^{2}}{4 \varepsilon_{0}}+\frac{4 \beta^{2}}{9 \varepsilon_{0}}\right)+\frac{9 \rho^{2}}{4 \varepsilon_{0}} \delta_{3}\right]\left\|s_{t}\right\|^{2} \\
& +\left[\delta_{1}\left(\varepsilon_{0}-1\right)+18 G \delta_{5}\right]\left\|s_{x}\right\|^{2}+\left[\delta_{1}\left(\varepsilon_{0}-\frac{4 \gamma}{3}\right)+36 \varepsilon_{0} \delta_{5}\right]\|s\|^{2} \\
& +\left[-\delta_{5} \rho+\varepsilon_{0} \delta_{1}+\varepsilon_{0} \delta_{3}\right]\left\|w_{t}\right\|^{2}+\left[\frac{\rho \delta_{3}}{2}-\frac{I_{\rho} \delta_{4}}{2}+\delta_{2} I_{\rho}\right]\left\|3 s_{t}-\psi_{t}\right\|^{2} \\
& +\left[\delta_{2}\left(\varepsilon_{0}-\frac{1}{2}\right)+4 \varepsilon_{0} \delta_{5}+2 G \delta_{5}\right]\left\|(3 s-\psi)_{x}\right\|^{2} \\
& +\left\{-\frac{G \delta_{3}}{2}+\frac{G^{2} \delta_{2}}{2}+\frac{G^{2} \delta_{4}}{2}+\delta_{5}\left(2 \varepsilon_{0}+\frac{3 G}{2}\right)\right\}\left\|\psi-w_{x}\right\|^{2}, \quad t \geq 0 .
\end{aligned}
$$

We shall forget for a moment about the first three terms on the right-hand side of (10) and focus on the rest of the coefficients. We need

$$
\left\{\begin{array}{l}
\delta_{1}\left(\varepsilon_{0}-1\right)+18 G \delta_{5}<0 \\
\delta_{1}\left(\varepsilon_{0}-\frac{4 \gamma}{3}\right)+36 \varepsilon_{0} \delta_{5}<0 \\
-\delta_{5} \rho+\varepsilon_{0} \delta_{1}+\varepsilon_{0} \delta_{3}<0 \\
\frac{\rho \delta_{3}}{2}-\frac{I_{\rho} \delta_{4}}{2}+\delta_{2} I_{\rho}<0 \\
\delta_{2}\left(\varepsilon_{0}-\frac{1}{2}\right)+4 \varepsilon_{0} \delta_{5}+2 G \delta_{5}<0 \\
-\frac{G \delta_{3}}{2}+\frac{G^{2} \delta_{2}}{2}+\frac{G^{2} \delta_{4}}{2}+\delta_{5}\left(2 \varepsilon_{0}+\frac{3 G}{2}\right)<0
\end{array}\right.
$$

Next, ignoring $\varepsilon_{0}$ as we will take it small enough later, we obtain

$$
\left\{\begin{array}{l}
18 G \delta_{5}<\delta_{1}, \\
\frac{\rho \delta_{3}}{2}+\delta_{2} I_{\rho}<\frac{I_{\rho} \delta_{4}}{2}, \\
2 G \delta_{5}<\frac{\delta_{2}}{2}, \\
\delta_{2} G+G \delta_{4}+3 \delta_{5}<\delta_{3} .
\end{array}\right.
$$


Observe that there is only a smallness condition on $\delta_{5}$, so we postpone its selection. There remains

$$
\left\{\begin{array}{l}
\frac{\rho \delta_{3}}{2}+\delta_{2} I_{\rho}<\frac{I_{\rho} \delta_{4}}{2}, \\
\delta_{2} G+G \delta_{4}<\delta_{3} .
\end{array}\right.
$$

In turn we see that there is only a smallness condition on $\delta_{2}$, therefore we need

$$
\left\{\begin{array}{l}
\rho \delta_{3}<I_{\rho} \delta_{4} \\
G \delta_{4}<\delta_{3}
\end{array}\right.
$$

These last relations (14) hold if $\rho<I_{\rho} / G$ by taking, for instance, $\delta_{4}=\left(\rho+\frac{I_{\rho}}{G}\right) \frac{\delta_{3}}{2 I_{\rho}}$. Now we go backward and select $\delta_{2}$ small enough (in terms of $\delta_{3}$ ) so that the relations in (13) are satisfied. Next we pick $\delta_{5}$ and then $\delta_{1}$ so that the remaining conditions (12) are fulfilled. At this stage we may choose $\varepsilon_{0}$ (small enough to satisfy (11)). Finally, we select $\delta_{3}$ so small that the three first coefficients in (10) be negative.

The inequality $F^{\prime}(t) \leq-\kappa F(t), t \geq 0$ implies the exponential decay of $F(t)$. This property is shared by $E(t)$ through the equivalence.

\section{Conclusion}

Our main goal here is the handling of the interfacial slip and the stabilization of the system. This has been established using a different way than the one used in the literature and with much weaker conditions. Indeed, the conditions $r_{1}:=\frac{G}{\rho} \neq \frac{D}{I_{\rho}}=: r_{2}$ and $k_{i} \neq r_{i}, i=1,2$, are dropped and replaced by $\rho G<I_{\rho}$.

\section{Competing interests}

The author declares that they have no competing interests.

\section{Acknowledgements}

The author would like to acknowledge the support provided by King Abdulaziz City for Science and Technology (KACST) through the Science and Technology Unit at King Fahd University of Petroleum and Minerals (KFUPM) for funding this work through project No. AC -32- 49.

Received: 1 June 2015 Accepted: 6 September 2015 Published online: 18 September 2015

\section{References}

1. Hansen, SW, Spies, R: Structural damping in a laminated beam due to interfacial slip. J. Sound Vib. 204, 183-202 (1997)

2. Beards, CF, Imam, IMA: The damping of plate vibration by interfacial slip between layers. Int. J. Mach. Tool Des. Res. 18, 131-137 (1978)

3. Feng, D, Shi, D, Zhang, W: Boundary feedback stabilization of Timoshenko beam with boundary dissipation. Sci. China Ser. A 41(5), 483-490 (1998)

4. Kim, JU, Renardy, Y: Boundary control of the Timoshenko beam. SIAM J. Control Optim. 25, 1417-1429 (1987)

5. Morgul, O: Boundary control of a Timoshenko beam attached to a rigid body: planar motion. Int. J. Control 54, 761-763 (1991)

6. Xu, GQ: Feedback exponential stabilization of a Timoshenko beam with both ends free. Int. J. Control 72(4), 286-297 (2005)

7. Xu, GQ, Feng, DX: The Riesz basis property of a Timoshenko beam with boundary feedback and application. IMA J. Appl. Math. 67, 357-370 (2002)

8. Xu, GQ, Feng, DX, Yung, SP: Riesz basis property of the generalized eigenvector system of a Timoshenko beam. IMA J. Math. Control Inf. 21(1), 65-83 (2004)

9. Xu, GQ, Yung, SP: Exponential decay rate for a Timoshenko beam with boundary damping. J. Optim. Theory Appl. 123(3), 669-693 (2004)

10. Yan, Q, Feng, D: Boundary stabilization of nonuniform Timoshenko beam with a tipload. Chin. Ann. Math. 22(4), 485-494 (2001)

11. Yan, QX, Hou, SH, Feng, DX: Asymptotic behavior of Timoshenko beam with dissipative boundary feedback. J. Math. Anal. Appl. 269, 556-577 (2002)

12. Zhang, CG: Boundary feedback stabilization of the undamped Timoshenko beam with both ends free. J. Math. Anal. Appl. 326, 488-499 (2007) 
13. Wang, JM, Guo, BZ: Analyticity and dynamic behavior of a damped three-layer sandwich beam. J. Optim. Theory Appl. 137(3), 675-689 (2008)

14. Wang, JM, Liu, J, Ren, B, Chen, J: Sliding mode control to stabilization of cascaded heat PDE-ODE systems subject to boundary control matched disturbance. Automatica 52, 23-34 (2015)

15. Wang, JM, Krstic, M: Stability of an interconnected system of Euler-Bernoulli beam and heat equation with boundary coupling. ESAIM Control Optim. Calc. Var. 21(4), 1029-1052 (2015)

16. Wang, JM, Xu, GQ, Yung, SP: Exponential stabilization of laminated beams with structural damping and boundary feedback controls. SIAM J. Control Optim. 44(5), 1575-1597 (2005)

17. Cao, XG, Liu, DY, Xu, GQ: Easy test for stability of laminated beams with structural damping and boundary feedback controls. J. Dyn. Control Syst. 13(3), 313-336 (2007)

Submit your manuscript to a SpringerOpen ${ }^{\circ}$ journal and benefit from:

- Convenient online submission

Rigorous peer review

- Immediate publication on acceptance

Open access: articles freely available online

- High visibility within the field

- Retaining the copyright to your article 